Rev. Int. Contam. Ambie. 34 (1) 93-102, 2018

DOI: 10.20937/RICA.2018.34.01.08

\title{
IMPACTO DE LA MINERÍA DEL ORO ASOCIADO CON LA CONTAMINACIÓN POR MERCURIO EN SUELO SUPERFICIAL DE SAN MARTÍN DE LOBA, SUR DE BOLÍVAR (COLOMBIA)
}

\author{
Leobardo ROCHA-ROMÁN, Jesús OLIVERO-VERBEL y Karina Rocío CABALLERO-GALLARDO*
}

Grupo de Química Ambiental y Computacional, Facultad de Ciencias Farmacéuticas, Universidad de Cartagena, Cartagena 130015, Colombia

*Autor de correspondencia; kcaballerog@unicartagena.edu.co

(Recibido octubre 2016; aceptado mayo 2017)

Palabras clave: toxicidad, ambiente, actividades antrópicas, salud humana

\section{RESUMEN}

Las actividades humanas han aumentado la contaminación por mercurio $(\mathrm{Hg})$ en los ambientes terrestres, especialmente en áreas de minería de oro. El objetivo de este estudio fue evaluar las concentraciones de mercurio total (Hg-t) en muestras de suelo superficial de San Martin de Loba, sur de Bolívar (Colombia), así como determinar el nivel de contaminación en el suelo. Un total de 202 muestras de suelo superficial fueron colectadas en San Martín de Loba. Los niveles de Hg-t fueron medidos por atomización electrotérmica y espectroscopia de absorción atómica. Las concentraciones de $\mathrm{Hg}$-t variaron de 0.002 a $23.83 \mu \mathrm{g} / \mathrm{g}$, con un valor medio de $3.40 \pm 0.36 \mu \mathrm{g} / \mathrm{g}$, el cual es ligeramente más alto que para el $\mathrm{Hg}$ en suelo a nivel mundial. El índice de geo-acumulación (Igeo) para suelo alcanzó valores superiores a 5, indicando una contaminación extrema (Clase 6) en el $17 \%$ de las muestras, mientras que el $70 \%$ fueron Clase 5 (fuertemente a extremadamente contaminado). Este estudio también ofrece un mapa de riesgos para evaluar las posibles repercusiones negativas. En conclusión, el municipio de San Martín de Loba puede considerarse una zona fuertemente contaminada con alto contenido de Hg. La tecnología utilizada para extraer oro a lo largo de los procesos de amalgamación provoca un alto grado de contaminación por $\mathrm{Hg}$ en torno a esta área de minería de oro. Por lo tanto, deben considerarse métodos alternativos de extracción para reducir los niveles de $\mathrm{Hg}$ que pueden liberarse al ambiente y afectar la salud humana.

Key words: toxicity, environment, anthropogenic activities, human health

\begin{abstract}
Human activities have increased mercury $(\mathrm{Hg})$ contamination of terrestrial environments, especially gold mining areas. The aim of this study was to evaluate total $\mathrm{Hg}$ concentrations (T-Hg) in surface soil samples of San Martín de Loba, south of Bolívar (Colombia), to determine their Hg contamination level. For that purpose, 202 surface soil samples were collected. T-Hg levels were measured by electrothermal atomization and atomic absorption spectroscopy. The T-Hg surface soil concentrations ranged between 0.002 and $23.83 \mu \mathrm{g} / \mathrm{g}$, with a median value of $3.40 \pm 0.36 \mu \mathrm{g} / \mathrm{g}$, which is
\end{abstract}


slightly higher than the international $\mathrm{Hg}$ soil levels. The geoaccumulation index ( $\left.\mathrm{I}_{\text {geo }}\right)$ for soils reached values greater than 5 , indicating extreme pollution (Class 6) in $17 \%$ of samples, while $70 \%$ was Class 5 (strongly to extremely polluted). This study also provides a risk map to evaluate potential negative repercussions. In conclusion, the municipality of San Martín de Loba can be considered as a strongly polluted area with high $\mathrm{Hg}$ content. Technology used to extract gold through amalgamation processes causes a high degree of $\mathrm{Hg}$ pollution around this gold mining area. Therefore, alternative extraction methods should be considered to reduce $\mathrm{Hg}$ levels that can be released into the environment and affect human health.

\section{INTRODUCCIÓN}

El mercurio $(\mathrm{Hg})$ es un elemento tóxico que se bioacumula y biomagnifica a través de la cadena trófica (Poste et al. 2015), constituyendo una amenaza para la salud humana (Li y Tse 2015). La contaminación por Hg puede deberse a fuentes naturales tales como volcanes, erosión del suelo y océanos, o bien a fuentes antrópicas tales como combustibles fósiles (Feng et al. 2002), producción de metales (Feng et al. 2004), minería del oro (Olivero-Verbel et al. 2015) y plantas de cloro-álcali (Qi et al. 2000), entre otras, siendo la minería aurífera la principal causa de contaminación ambiental por este metal debido a la utilización de mercurio metálico para formar amalgamas con el oro durante la extracción del oro (Niane et al. 2014).

$\mathrm{La}$ amalgamación con $\mathrm{Hg}$ se utiliza indiscriminadamente en la recuperación de oro en la minería artesanal en el sur del departamento de Bolívar. El Hg es liberado al ambiente a través de las aguas residuales, las colas de la flotación también conocidas como relaves y el vapor generado durante la quema de la amalgama para separar el oro. Este metal posee una alta presión de vapor, por lo que puede vaporizarse fácilmente a la atmósfera y condensarse en partículas en la misma. El Hg puede depositarse en suelos por efecto del lavado del agua de lluvia o el rocío, y afectar a la flora y fauna, e inclusive a la población humana (Adjorlolo-Gasokpoh et al. 2012).

La minería de oro artesanal y a pequeña escala es una fuente de subsistencia en muchos países (Santos-Francés et al. 2011), así como un factor determinante para la degradación del ambiente. Una vez que el $\mathrm{Hg}$ elemental entra en los ecosistemas acuáticos puede convertirse en metilmercurio (forma más tóxica del metal) por la acción bacteriana (Fleming et al. 2006). De esta forma es acumulado por la fauna acuática, principalmente por peces depredadores, cuyo consumo es una de las más importantes fuentes de riesgo para la salud de las poblaciones ribereñas, para quienes los productos de la pesca constituyen la principal fuente de proteínas (Olivero-Verbel et al. 2011).

En Colombia, de acuerdo con el Sistema de Información Minero Colombiano (SIMCO) para el año 2016, Bolívar fue el cuarto departamento con la mayor producción de oro (4084.01 kg) y San Martín de Loba el sexto municipio con la mayor producción de este mineral (104.08 kg) (SIMCO 2017). Este tipo de minería representa el $0.1 \%$ de la producción anual de oro (MINAMBIENTE 2015). En la minería aurífera realizada en el país se emplea mercurio metálico (Cordy et al. 2011), lo que ha ocasionado algunos impactos en la vegetación, degradación del suelo y contaminación de los recursos hídricos.

$\mathrm{El} \mathrm{Hg}$ puede aparecer de forma natural en el suelo, aunque en concentraciones muy bajas o como resultado de las actividades humanas. Además, es uno de los reservorios más importantes de este metal al ser liberado al ambiente, lo cual puede proporcionar un registro de su deposición. El contenido de $\mathrm{Hg}$ a nivel mundial en suelos es de alrededor de $0.06 \mu \mathrm{g} / \mathrm{g}$ (Berrow y Reaves 1984). En suelos contaminados con $\mathrm{Hg}$, su contenido depende en gran medida de las fuentes contaminantes como la minería de cinabrio, la metalúrgica (Llanos et al. 2011), la extracción de oro (Kpan et al. 2014) y la industria química (Miller et al. 2013), entre otras. Varios estudios a nivel mundial han reportado concentraciones de $\mathrm{Hg}$ en suelos, principalmente proveniente de minería aurífera, entre otros en Kenia (Odumo et al. 2014), China (Chen et al. 2016) y Venezuela (Santos-Francés et al. 2011).

Por otra parte, el impacto de la minería artesanal del oro en Colombia debido a la utilización de $\mathrm{Hg}$ se ha reportado en diferentes muestras de aire (Olivero-Verbel et al. 2014), peces (Olivero y Solano 1998, Marrugo-Negrete et al. 2008a), sedimentos y macrófitas (Olivero-Verbel et al. 2015), cabello humano (Olivero-Verbel et al. 1995, 2011, 2015), agua (Olivero-Verbel et al. 2015), fitoplancton y zooplancton (Marrugo-Negrete et al. 2008b), y animales 
domésticos (gallinas, cerdos y patos) (Argumedo et al. 2013), principalmente en los departamentos de Sucre (Olivero-Verbel et al. 2002, MarrugoNegrete et al. 2008a), Antioquía (Cordy et al. 2011) y Bolívar (Olivero-Verbel et al. 2011, 2014, 2015). Sin embargo, a pesar de la amplia información que existe sobre los efectos de la minería en el país, la mayoría de las investigaciones han sido relacionadas con otras matrices, y son pocos los estudios en suelo. Recientemente, García et al. (2015) evaluaron los niveles de $\mathrm{Hg}$ en suelos y lechos de quebradas en la zona minera de Miraflores, Quinchía, Colombia, encontrando un promedio de $\mathrm{Hg}$ en el suelo de 7.1 $\pm 6.2 \mu \mathrm{g} / \mathrm{g}$ entre 0 y $25 \mathrm{~cm}$ de profundidad y $8.9 \pm$ $7.9 \mu \mathrm{g} / \mathrm{g}$ entre 25 y $50 \mathrm{~cm}$, con un valor mínimo de $0.11 \mu \mathrm{g} / \mathrm{g}$ y máximo de $36.9 \mu \mathrm{g} / \mathrm{g}$. En el caso de San Martín de Loba se han realizado estudios en cabello humano (Olivero-Verbel et al. 1995, 2011); peces, sedimentos, agua y macrófitas (Olivero-Verbel et al. 2015), y aire (Olivero-Verbel et al. 2014), pero ninguna investigación ha determinado este metal en suelo.

El contenido de metales como $\mathrm{Hg}$ en suelo resulta tanto de fuentes naturales como antrópicas, siendo estas últimas las de mayor contribución. Varios estudios han reportado niveles de este elemento en suelo superficial por diferentes fuentes tales como la industria (Loska et al. 2004, Yaylali-Abanuz 2011) y la minería (Kocman et al. 2004). Las actividades antrópicas son las que provocan mayor acumulación, la cual puede ser varias veces superior al contenido en áreas no contaminadas. No obstante, en áreas distantes a los sitios de contaminación también se ha encontrado el metal debido al transporte atmosférico (Fu et al. 2010). Muchos factores ambientales pueden interferir con el proceso de adsorción/desorción del Hg, entre ellos especiación de $\mathrm{Hg}, \mathrm{pH}$ del suelo, iones cloruro, contenido de materia orgánica, forma y contenido de coloides en el suelo, y iones inorgánicos competitivos (Jing et al. 2007). El cinabrio ( $\mathrm{HgS})$ y mercurio elemental $\left(\mathrm{Hg}^{\circ}\right)$ son las principales fuentes naturales de mercurio en el suelo y en los ambientes acuáticos. Dependiendo de las condiciones redox, el $\mathrm{Hg}$ inorgánico puede aparecer en varios estados: $\mathrm{Hg}_{2}{ }^{2+}, \mathrm{Hg}^{\circ} \mathrm{y} \mathrm{Hg}^{2+}$. Los dos últimos se encuentran normalmente en el suelo.

$\mathrm{El} \mathrm{Hg}$ es considerado un metal pesado de gran importancia como contaminante ambiental, ya que debido a su toxicidad ha ocasionado muchos episodios de intoxicaciones humanas (Tsubaki y Irukayama 1977, Taueg et al. 1992, Wößmann et al. 1999, Korbas et al. 2010). Además, contamina el suelo y el agua tanto superficial como subterránea, por lo que el monitoreo en zonas donde se utiliza es fundamental. Hasta la fecha, el impacto del $\mathrm{Hg}$ en suelos ha sido poco estudiado aunque representa el sumidero más importante y por lo tanto también es una fuente del metal en el ambiente. Con el objeto de ampliar el conocimiento sobre la presencia de $\mathrm{Hg}$ en el suelo debido a la minería de oro en Colombia, en este trabajo se determinaron los niveles de $\mathrm{Hg}$ en suelos del municipio de San Martín de Loba en el sur del departamento de Bolívar, una de las principales zonas de minería del oro en el país.

\section{MATERIALES Y MÉTODOS}

\section{Área de estudio}

El área de estudio está localizada en el departamento de Bolívar, Colombia, en el municipio de San Martín de Loba (SML), el cual está ubicado al norte del país y al sureste del departamento de Bolívar, a $8^{\circ} 56^{\prime} 37^{\prime}$ " N y $74^{\circ} 02^{\prime} 30^{\prime}$ ' S. Tiene una superficie de $414 \mathrm{~km}^{2}$ y es parte de la cuenca de la región momposina en el margen sur del Brazo de Loba del río Magdalena. Limita al norte con Hatillo de Loba y Brazo de Loba, al sur con Río Viejo y parte de Regidor, al oeste con Barranco de Loba y Ciénaga de Santa Rosa, y al este con Peñón Bolívar y parte de Brazuelo de Papayal. La cabecera municipal de SML está localizada en el margen sur del Brazo de Loba del río Magdalena, encontrándose a 15 min por vía fluvial del municipio de El Banco Magdalena y a una distancia aproximada de $445 \mathrm{~km}$ de la capital del departamento, Cartagena. Tiene una temperatura media de $30^{\circ} \mathrm{C}$. La base de la economía de San Martín de Loba son las actividades agrícola, pecuaria, pesquera, comercial y minera, siendo esta última la principal fuente de contaminación por $\mathrm{Hg}$ en el municipio de SML debido a la utilización de tecnologías muy rudimentarias (Alcaldía SMDL 2015).

El clima de esta región es tropical. La lluvia es significativa la mayor parte del año y la corta estación seca tiene poco efecto. La temperatura promedio es de $28.3^{\circ} \mathrm{C}$ y la precipitación de aproximadamente $2201 \mathrm{~mm}$. El mes más seco corresponde a enero, con $20 \mathrm{~mm}$ de lluvia. La mayor parte de la precipitación en este municipio ocurre en octubre, con $378 \mathrm{~mm}$. Por su parte, hay una diferencia de $358 \mathrm{~mm}$ entre los meses más secos y los más húmedos. A lo largo del año, las temperaturas varían en $1.6^{\circ} \mathrm{C}$ (Datos Climáticos Mundiales 2017).

\section{Colección de muestras y preparación}

Se colectaron un total de 202 muestras (300 g) de suelo superficial en la cabecera municipal de 
SML. Se colocaron en recipientes plásticos estériles previamente rotulados y almacenados en neveras de espuma de polietileno con hielo hasta llegar al Laboratorio del Grupo de Investigación en Química Ambiental y Computacional de la Universidad de Cartagena, Colombia, donde se almacenaron a -20 ${ }^{\circ} \mathrm{C}$ hasta su procesamiento. En el laboratorio las muestras de suelo fueron secadas y homgeneizadas con un mortero de porcelana (Adjorlolo-Gasokpoh et al. 2012), y tamizadas para obtener un tamaño de partícula $<1 \mathrm{~mm}$.

\section{Determinación de mercurio total (Hg-t)}

Para determinar la concentración de Hg-t en suelo superficial se pesaron $5 \mathrm{mg}$ de muestra seca en una balanza analítica Ohaus con precisión de \pm $0.1 \mathrm{mg}$ y se colocaron en un portamuestras para la cuantificación de $\mathrm{Hg}$-t mediante pirólisis a $800{ }^{\circ} \mathrm{C}$, seguido de espectrometría de absorción atómica Zeeman diferencial a través de un analizador de Hg Lumex RA-915+ (Sholupov et al. 2004). Para la determinación de este elemento, los dos métodos de espectroscopia de absorción atómica más utilizados han sido los de cámara de Delves y de horno de grafito. Este último (análisis por absorción atómica con atomización electrotérmica con horno de grafito) tiene múltiples ventajas, debido a que en la actualidad puede ser fácilmente automatizado y es un método simple, cuya precisión y exactitud son elevadas y similares a los del procedimiento de la cámara de Delves.

La determinación de los niveles de $\mathrm{Hg}$ total en las muestras de suelo superficial se realizó mediante el método de estándar externo. Para este propósito varias cantidades de muestra certificada se pesaron en una balanza analítica de laboratorio Ohaus con precisión de $\pm 0.1 \mathrm{mg}$ y se depositaron en los portamuestras del equipo para el análisis directo de $\mathrm{Hg}$ total y su respectiva lectura. Una vez obtenidos los valores de absorbancia y la masa del estándar de referencia, se construyeron curvas de calibración, teniendo como criterio aquellas en que los valores de coeficiente de determinación $\left(\mathrm{R}^{2}\right)$ fueron cercanos a 1 (Olivero-Verbel et al. 2009). La exactitud del método se evaluó mediante muestra certificada para sedimento (IAEA-433). La exactitud obtenida fue de $98.10 \%$, teniendo en cuenta que la concentración media fue de $0.167 \pm 0.10 \mu \mathrm{g} / \mathrm{g}$ frente a un valor certificado de $0.168 \pm 0.017 \mu \mathrm{g} / \mathrm{g}$. La precisión se determinó mediante el cálculo del coeficiente de variación $(\mathrm{CV})$. Todos los valores de $\mathrm{CV}$ entre duplicados de la misma muestra fueron inferiores al 10 $\%$, obteniéndose datos entre 0 y $9 \%$. La base para calcular el límite de detección (LD) fue tres veces la desviación estándar de los blancos. E1 LD para mercurio total en suelo fue de $0.002 \mu \mathrm{g} / \mathrm{g}$.

\section{Determinación de contaminación de suelo}

El índice de geoacumulación (Igeo) fue empleado para determinar el nivel de contaminación por $\mathrm{Hg}$ en suelo (Müller 1981):

$\mathrm{Igeo}_{\mathrm{ge}}=\log _{2}[\mathrm{Cn} / \mathrm{ABn}]$

donde Cn es la concentración del metal en suelo, $A$ es una constante (1.5) y $B n$ es el valor de fondo geoquímico del metal, que para este estudio es de $0.06 \mu \mathrm{g} / \mathrm{g}$ (Berrow y Reaves 1984). La contaminación por Hg se clasifica en: Clase 0 (no contaminado): Igeo $\leq 0$; Clase 1 (no contaminado a moderadamente contaminado): 0 $\leq$ Igeo $\leq 1$; Clase 2 (contaminación moderada): $1 \leq$ Igeo $_{\text {ge }}$ $\leq 2$; Clase 3 (contaminación moderada a fuertemente contaminada): $2 \leq$ Igeo $\leq 3$; Clase 4 (fuertemente contaminada): $3 \leq$ Igeo $\leq 4$; Clase 5 (fuertemente a extremadamente contaminado): $4 \leq$ Igeo $\leq 5$; Clase 6 (extremadamente contaminado): Igeo $>5$.

\section{Análisis de datos}

Los resultados de las determinaciones de $\mathrm{Hg}$ total en suelo superficial se presentan como la media \pm el error estándar, el cual fue calculado teniendo en cuenta la ecuación:

Error estándar $=S /$ raíz cuadrada de $(n)$

donde: $S$ es la desviación estándar y $n$ es el tamaño (número de individuos de la muestra).

\section{RESULTADOS}

El promedio de la media de las concentraciones de Hg-t en suelo superficial en el municipio de SML fue de $3.40 \pm 0.36 \mu \mathrm{g} / \mathrm{g}$. El intervalo fue de $0.002 \mathrm{a}$ $23.83 \mu \mathrm{g} / \mathrm{g}$. Los resultados por punto de muestreo fueron agrupados teniendo en cuenta los valores de $\mathrm{Hg}$ en suelo reportados a nivel mundial, los cuales se muestran en el cuadro I. De acuerdo con estos resultados, $93 \%$ de los puntos de muestreo son superiores a los valores para suelo en países como Eslovenia, Alemania, Canadá, Estados Unidos y China, así como a nivel mundial, en tanto que el $7 \%$ fue encontrado por debajo de los valores de fondo observados para el Reino Unido.

Para la representación de los niveles de Hg-t en suelo superficial del municipio de SML, los datos 
CUADRO I. VALORES DE FONDO PARA Hg EN SUELO REPORTADOS EN OTROS PAÍSES

\begin{tabular}{lcl}
\hline Lugar & $\begin{array}{r}\text { Concentración } \\
\text { de } \begin{array}{l}\text { Hg total } \\
(\mu \mathrm{g} / \mathrm{g})\end{array}\end{array}$ & \\
\hline Mundial & 0.06 & Berrow y Reaves (1984) \\
Reino Unido & 0.03 & Davies (1976) \\
Eslovenia & 0.07 & Sajn (2003) \\
Alemania & 0.09 & Kick et al. 1980) \\
Canadá & 0.06 & McKeague y Wolynetz (1980) \\
Estados & & Shacklette y Boerngen (1984) \\
Unidos & 0.09 & Zhang et al. (2008) \\
China & 0.06 & \\
\hline
\end{tabular}

fueron agrupados como aparecen en el cuadro II, teniendo en cuenta los valores de fondo reportados en la literatura (Berrow y Reaves 1984). Es importante destacar que la definición de concentraciones de línea base para metales pesados en suelo es esencial para reconocer el estado de la contaminación en éstos. Sin embargo, la determinación de los niveles de fondo para estos elementos en el suelo es compleja y puede variar naturalmente en varios órdenes de magnitud (Hamon et al. 2004). Los datos muestran que solamente 14 muestras correspondientes a $7 \%$ del total, se encuentran clasificadas como no contaminadas de acuerdo con el Igeo. No obstante, los resultados para la mayoría de las muestras se consideran indicativos de alta contaminación, lo cual demuestra que la actividad minera tiene un impacto sobre la contaminación por $\mathrm{Hg}$ del suelo superficial y muy probablemente sobre la población del municipio de SML. De acuerdo con lo reportado previamente por Olivero-Verbel et al. (2015), el destino ambiental del $\mathrm{Hg}$ en el distrito minero de San Martín de Loba se debe principalmente a la minería del oro por el uso del metal en los procesos de extracción. En el caso de San Martín de Loba, los seres humanos que viven en esta zona urbana se ven afectados cuando el $\mathrm{Hg}$ es liberado a la atmósfera debido a los procesos de quema de amalgama, o directamente por el suelo cuando los mineros recorren las calles con zapatos contaminados con residuos mineros.

Con los datos de Hg-t en suelo superficial y las coordenadas geográficas de cada punto fue posible construir un mapa en el cual se representa el grado de contaminación en el municipio de SML, teniendo en cuenta la clasificación del cuadro II. La ubicación de las 202 muestras de suelo superficial se presenta en la figura 1. La zona con el valor más alto (23.83 $\pm 0.33 \mu \mathrm{g} / \mathrm{g}$ ) correspondió a un antiguo pozo minero abandonado que en la actualidad es una zona residencial. Además, en esa zona también se han reportado niveles altos de $\mathrm{Hg}$ en aire debido a la presencia de entables mineros (minas artesanales). Sin embargo, también fueron identificados otros puntos con altas concentraciones de $\mathrm{Hg}$ en zonas de mayor afluencia de mineros como cantinas y tiendas de variedades, incluyendo las minas abandonadas o en actividad ubicadas dentro del municipio de San Martín de Loba.

\section{DISCUSIÓN}

En comparación con las concentraciones de $\mathrm{Hg}$ en suelos de otras áreas (cuadro III), la media de este estudio fue más baja que los niveles reportados en Venezuela (Santos-Francés et al. 2011) y en las zonas mineras de Miraflores, Quinchía, Colombia (García et al. 2015). Sin embargo, cuando se compararon con suelos de China (Chen et al. 2016) fueron más altos. Las concentraciones muy elevadas de $\mathrm{Hg}$ podrían explicarse en términos del proceso de recuperación

CUADRO II. NIVELES DE Hg TOTAL EN SUELO SUPERFICIAL AGRUPADOS DE ACUERDO CON LOS VALORES DE REFERENCIA

\begin{tabular}{lcccccc}
\hline Grupo & $\mathrm{n}$ & Nivel de Hg total $(\mu \mathrm{g} / \mathrm{g})$ & Intervalo & Igeo & Clase Igeo & Clasificación \\
\hline$<0.06$ & 14 & $0.01 \pm 0.001$ & $0.002-0.01$ & 0 & 0 & No contaminado \\
\hline$>0.06 \mathrm{y}<0.3$ & 12 & $0.16 \pm 0.02$ & $0.10-0.29$ & 0.8 & $\begin{array}{l}\text { No contaminado } \\
\text { a moderadamente } \\
\text { contaminado }\end{array}$ \\
\hline$>0.3 \mathrm{y}<5.0$ & 141 & $1.57 \pm 0.10$ & $0.32-4.83$ & 4.1 & 5 & $\begin{array}{l}\text { Fuertemente a } \\
\text { extremadamente } \\
\text { contaminado }\end{array}$ \\
\hline$>5 \mu \mathrm{g} / \mathrm{g}$ & 35 & $13.24 \pm 0.90$ & $5.31-23.83$ & 7.2 & 6 & $\begin{array}{l}\text { Extremadamente } \\
\text { contaminado }\end{array}$ \\
\hline
\end{tabular}



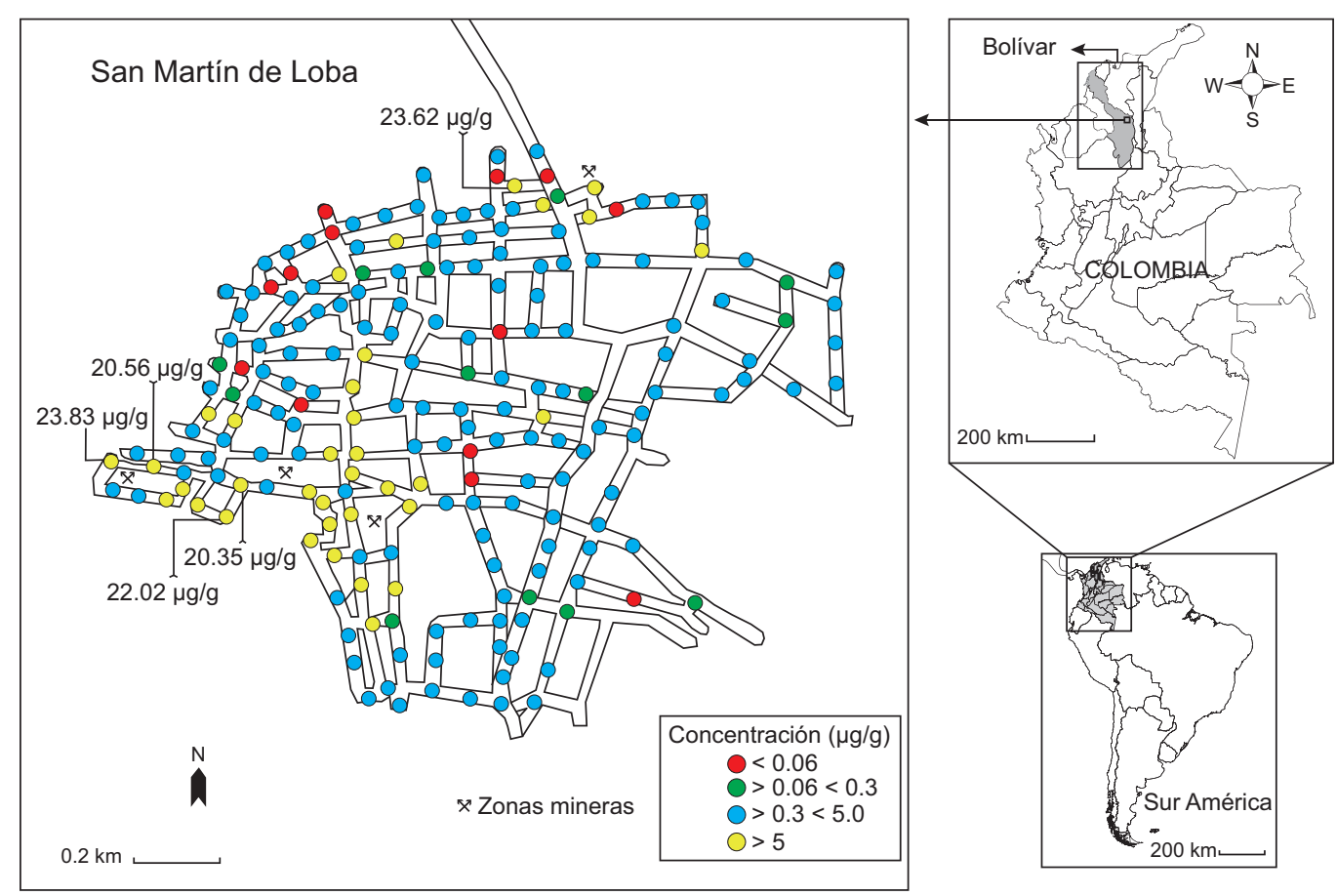

Fig. 1. Contaminación ambiental por Hg en suelo superficial en la cabecera municipal de San Martín de Loba, sur de Bolívar (Colombia)

CUADRO III. CONCENTRACIONES DE Hg EN SUELO PROVENIENTES DE ZONAS DE MINERÍA AURÍFERA REPORTADOS EN LA LITERATURA

\begin{tabular}{lcccl}
\hline Lugar & $\begin{array}{c}\text { Profundidad } \\
(\mathrm{cm})\end{array}$ & $\begin{array}{c}\text { Concentración } \\
\text { promedio }(\mu \mathrm{g} / \mathrm{g})\end{array}$ & Intervalo & Referencia \\
\hline China & $0-20$ & 9.57 & $0.06-63.79$ & Chen et al. (2016) \\
Kenia & $0-30$ & 0.14 & $0.02-1.10$ & Odumo et al. (2014) \\
Colombia & $0-25$ & 7.1 & $0.11-36.90$ & García et al. (2015) \\
& $25-50$ & 8.9 & & \\
Venezuela & $0-10$ & 26.89 & $0.16-542$ & Santos-Francés et al. (2011) \\
Colombia & $0-20$ & 3.4 & $0.002-23.83$ & Este estudio \\
\hline
\end{tabular}

de oro durante la amalgamación con $\mathrm{Hg}$, ya que durante la quema de la amalgama este elemento pasa a la atmósfera y posteriormente ocurre su deposición seca y húmeda.

La concentración de Hg-t en la mayoría de los suelos en las zonas mineras estudiadas puede considerarse crítica. De acuerdo con Kabata-Pendias (2001), el contenido crítico de $\mathrm{Hg}$ en los suelos es de $0.3-5 \mu \mathrm{g} / \mathrm{g}$. Con base en este límite, $70 \%$ de los suelos muestreados en el municipio de SML contienen niveles más altos y $12 \%$ de las muestras presentan concentraciones de $\mathrm{Hg}$ por arriba de $10 \mu \mathrm{g} / \mathrm{g}$, por lo que se clasifican como altamente contaminadas.

El índice de geo-acumulación (Igeo) (Müller 1981) y el índice de carga de contaminación (PLI) (Tomlinson et al. 1980) han sido empleados en la determinación de la contaminación en suelos por metales pesados (Howari et al. 2004, Bhuiyan et al. 2010, Mmolawa et al. 2011). El Igeo es la medida cuantitativa del grado de contaminación del metal, la cual divide en siete clases. Por su parte, el índice de carga de contaminación es una herramienta rápida cuyo objetivo es comparar el estado de contaminación de diferentes lugares; se utiliza para determinar la gravedad de la contaminación y su variación a lo largo de la muestra en diferentes estaciones (Rabee et al. 2011). Por lo tanto, la concentración basal geoquímica es más útil porque estadísticamente es el $95 \%$ del rango esperado de concentraciones de fondo (Gough et al. 1994, Chen et al. 1999).

El valor de fondo geoquímico o natural es una medida relativa para diferenciar las concentraciones de elementos naturales o compuestos de las concentraciones 
antrópicas en una muestra ambiental (Matschullat et al. 2000). Se han realizado varios estudios en diferentes regiones del mundo para estimar el valor de fondo geoquímico y la concentración de línea base de elementos traza (Gough et al. 1994, Tobías et al. 1997, Chen et al. 1999, Zhao et al. 2007, Bini et al. 2011). De acuerdo con lo expuesto, en este estudio se calculó el Igeo como una herramienta de calidad para determinar el grado de contaminación por metales, en este caso Hg. Para tal fin, y como no se disponía de valores de línea base (valores locales de áreas no contaminadas), se utilizó como nivel de fondo de la concentración de $\mathrm{Hg}$ en suelo a nivel mundial $(0.06 \mu \mathrm{g} / \mathrm{g})$ (Berrow y Reaves 1984). Se encontró que el $87 \%$ de los valores calculados para Igeo fueron $>4$, lo que indica que los suelos se clasifican como fuertemente contaminados con $\mathrm{Hg} ; 6 \%$ tuvo un valor de $\mathrm{I}_{\text {geo }} \leq 1$, por lo que estos suelos se categorizaron como no contaminados a moderadamente contaminados, y el $7 \%$ correspondió a los no contaminados. Estos resultados son comparables a los reportados por Santos-Francés et al. (2011), quienes encontraron valores de $\mathrm{I}_{\text {geo }}>3$ en Hg superficial en Venezuela. Es claro que las concentraciones más altas de Hg-t en suelo detectadas en este estudio están dentro de los intervalos reportados en otras investigaciones para áreas de minería de oro.

Actualmente han aumentado las concentraciones de $\mathrm{Hg}$ en suelos debido principalmente a la quema de combustibles fósiles combinados con procesos de transporte atmosférico de largo alcance. Otras fuentes como las plantas de cloro-álcali, la minería del oro y la producción de cemento también pueden ser significativas, al menos a nivel local (Xu et al. 2015). Se observa que el sitio donde se encontraron los valores más altos de $\mathrm{Hg}$-t $(>10 \mu \mathrm{g} / \mathrm{g})$ en suelo en el municipio de SML coincide con el sitio donde Olivero-Verbel et al. (2014) reportaron concentraciones de $\mathrm{Hg}$-t en aire superiores a $200 \mathrm{ng} / \mathrm{m}^{3}$. En esta área funcionaban y todavía están en actividad minas artesanales (entables mineros) y los niveles de $\mathrm{Hg}$ en aire reportados por estos autores son $>10000 \mathrm{ng} / \mathrm{m}^{3}$. Lo anterior sustenta los niveles mayores de $10 \mu \mathrm{g} / \mathrm{g}$ encontrados en este estudio, lo que pone en evidencia que la actividad minera fue y sigue siendo la fuente de la contaminación en esta área.

Por otra parte, se ha reportado que el suelo se contamina con $\mathrm{Hg}$ principalmente por la deposición húmeda y seca de $\mathrm{Hg}^{2+} \mathrm{y} \mathrm{Hg}^{0}$, así como por la sedimentación de partículas de este metal (Biester et al. 2002. Teniendo en cuenta lo expuesto anteriormente, es muy probable que el $\mathrm{Hg}$, asociado con la deposición húmeda y seca, sea uno de los mecanismos por los cuales el Hg se transporta y acumula en el suelo de las calles de SML. No obstante, el transporte directo del metal en las botas contaminadas con sedimentos que lo contienen, constituye igualmente un proceso de dispersión del contaminante a lo largo de esta localidad. Este último factor podría controlarse si los mineros cambiaran por completo su ropa de trabajo antes de abandonar la mina y dirigirse a sus viviendas. Además, es importante señalar que los sitios mineros son puntos críticos de contaminación por $\mathrm{Hg}$ en suelo (Yin et al. 2016).

\section{CONCLUSIONES}

Se determinó un alto contenido de Hg-t en suelo superficial proveniente del municipio de San Martín de Loba, una zona de influencia de minería aurífera. La tecnología utilizada para extraer oro a lo largo de los procesos de amalgamación ha sido y es causa de la contaminación por $\mathrm{Hg}$ en el área de influencia de la operación minera.

Los resultados revelan que altos niveles de $\mathrm{Hg}$ están presentes a la entrada del municipio, cerca de las minas, tanto de aquellas que han sido abandonadas como de las que siguen activas, así como en tiendas de oro, entre otros.

Este trabajo aporta el primer mapa de contaminación por Hg-t en suelo superficial en el municipio de SML, mostrando que es una zona impactada por el $\mathrm{Hg}$ asociado a la actividad minera. Por lo tanto, es necesario implementar métodos alternativos para la extracción de oro y posiblemente realizar actividades de remediación de suelos que pueden variar desde el confinamiento y la estabilización hasta el uso de una cubierta vegetal o con minerales libres de $\mathrm{Hg}$, de tal forma que este metal no constituya un riesgo potencial para la salud de los habitantes de SML.

\section{AGRADECIMIENTOS}

Los autores dan las gracias al programa de formación doctoral de Colombia (Colciencias, 567-2012).

\section{REFERENCIAS}

Adjorlolo-Gasokpoh A., Golow A.A. y Kambo-Dorsa J. (2012). Mercury in the surface soil and cassava, Manihot esculenta (flesh, leaves and peel) near gold mines at Bogoso and Prestea, Ghana. Bull. Environ. Contam. Toxicol. 89 (6), 1106-1110.

DOI: $10.1007 / \mathrm{s} 00128-012-0849-7$ 
Alcaldía SMDL (2015). Nuestro Municipio. Alcaldía de San Martín de Loba-Bolívar. [en línea]. http://www. sanmartindeloba-bolivar.gov.co/informacion general. shtml 12/03/2017.

Argumedo M., Vidal J. y Marrugo J.L. (2013). Mercurio total en animales domésticos en mina Santa Cruz, sur de Bolívar-Colombia. RECIA 5 (2), 366-379.

Berrow M.L. y Reaves G.A. (1984). Background of trace elements in soils. Memorias.. 1st International Conference on Environmental Contamination. CEP Consultants. Edimburgo, Reino Unido, 333-340.

Bhuiyan M.A.H., Parvez L., Islam M.A., Dampare S.B. y Suzuki S. (2010). Heavy metal pollution of coal mine-affected agricultural soils in the northern part of Bangladesh. J. Hazard. Mater. 173 (1-3), 384-392. DOI: 10.1016/j.jhazmat.2009.08.085

Biester H., Müller G. y Schöler H.F. (2002). Binding and mobility of mercury in soils contaminated by emissions from chlor-alkali plants. Sci. Total Environ. 284 (1-3), 191-203. DOI: 10.1016/S0048-9697(01)00885-3

Bini C., Sartori G., Wahsha M. y Fontana S. (2011). Background levels of trace elements and soil geochemistry at regional level in NE Italy. J. Geochem. Explor. 109 (1-3), 125-133. DOI: 10.1016/j.gexplo.2010.07.008

Cordy P., Veiga M.M., Salih I., Al-Saadi S., Console S., García O., Mesa L.A., Velásquez-López P.C. y Roeser M. (2011). Mercury contamination from artisanal gold mining in Antioquia, Colombia: The world's highest per capita mercury pollution. Sci. Total Environ. 410411, 154-160. DOI: 10.1016/j.scitotenv.2011.09.006

Chen M., Ma L.Q. y Harris W.G. (1999). Baseline concentrations of 15 trace elements in Florida surface soils. J. Environ. Qual. 28 (4), 1173-1181. DOI: $10.2134 /$ jeq1999.00472425002800040018x

Chen X., Ji H., Yang W., Zhu B. y Ding H. (2016). Speciation and distribution of mercury in soils around gold mines located upstream of Miyun Reservoir, Beijing, China. J. Geochem. Explor. 163, 1-9.

DOI: 10.1016/j.gexplo.2016.01.015c

Davies B.E. (1976). Mercury content of soils in western Britain with special reference to contamination from base metal mining. Geoderma 16 (3), 183-192.

DOI: 10.1016/0016-7061(76)90020-3

Datos Climáticos Mundiales (2017). Clima: San Martín de Loba. [en línea]. https://es.climate-data.org/location/50108/

Feng X., Sommar J., Lindqvist O. y Hong Y. (2002). Occurrence, emissions and deposition of mercury during coal combustion in the province Guizhou, China. Water Air Soil Poll. 139 (1), 311-324. DOI: $10.1023 / \mathrm{A}: 1015846605651$

Feng X., Li G. y Qiu G. (2004). A preliminary study on mercury contamination to the environment from artisanal zinc smelting using indigenous methods in Hezhang county, Guizhou, China-Part 1: mercury emission from zinc smelting and its influences on the surface waters. Atmos. Environ. 38 (36), 6223-6230. DOI: 10.1016/j.atmosenv.2004.07.020

Fleming E.J., Mack E.E., Green P.G. y Nelson D.C. (2006). Mercury methylation from unexpected sources: Molybdate-inhibited freshwater sediments and an iron-reducing bacterium. Appl. Environ. Microbiol. 72 (1), 457-464. DOI: 10.1128/AEM.72.1.457-464.2006

Fu X., Feng X., Zhu W., Rothenberg S., Yao H. y Zhang H. (2010). Elevated atmospheric deposition and dynamics of mercury in a remote upland forest of southwestern China. Environ. Pollut. 158 (6), 2324-2333.

DOI: $10.1016 /$ j.envpol.2010.01.032

García J., Muñoz D.A. y Morales J. (2015). Evaluación del contenido de mercurio en suelos y lechos de quebradas en la zona minera de Miraflores, Quinchía, Colombia. Acta Agronómica 64 (2), 165-177. DOI: 10.15446/acag.v64n2.40639

Gough L.P., Severson R.C. y Jackson L.L. (1994). Baseline element concentrations in soils and plants, Bull Island, Cape Romain national wildlife refuge, South Carolina, USA. Water Air Soil Poll. 74 (1), 1-17.

DOI: $10.1007 / \mathrm{BF} 01257145$

Hamon R.E., McLaughlin M.J., Gilkes R.J., Rate A.W., Zarcinas B., Robertson A., Cozens G., Radford N. y Bettenay L. (2004). Geochemical indices allow estimation of heavy metal background concentrations in soils. Global Biogeochem. Cy. 18 (1), 1-6.

DOI: 10.1029/2003GB002063

Howari F.M., Abu-Rukah Y. y Goodell P.C. (2004). Heavy metal pollution of soils along north Shuna-Aqaba Highway, Jordan. Inter. J. Environ. Pollut. 22 (5), 597607. DOI: $10.1504 /$ IJEP.2004.005913

Jing Y.D., He Z.L. y Yang X.E. (2007). Effects of pH, organic acids, and competitive cations on mercury desorption in soils. Chemosphere 69 (10), 1662-1669. DOI: 10.1016/j.chemosphere.2007.05.033

Kabata-Pendias A. (2001). Trace elements in soils and plants. 3rd ed. CRC Press, 413 pp.

Kick H., Burger H. y Sommer K. (1980). Gesamtgehalte an $\mathrm{Pb}, \mathrm{Zn}, \mathrm{Sn}, \mathrm{As}, \mathrm{Cd}, \mathrm{Hg}, \mathrm{Cu}, \mathrm{Ni}, \mathrm{Cr}$ and $\mathrm{Co}$ in landwirtschaftlich und gartnerisch genutzten Boden Nordrhein-Westfalens. Landwirtschaftliche Forschung [en línea]. http://agris.fao.org/agris-search/search. do?recordID=US201302829120 29/04/2017.

Kocman D., Horvat M. y Kotnik J. (2004). Mercury fractionation in contaminated soils from the Idrija mercury mine region. J. Environ. Monit. 6 (8), 696-703. DOI: $10.1039 / \mathrm{B} 403625 \mathrm{E}$

Korbas M., O’Donoghue J., Watson G.E., Pickering I.J., Singh S.P., Myers G.J., Clarkson T.W. y George G.N. 
(2010). The chemical nature of mercury in human brain following poisoning or environmental exposure. ACS Chem. Neurosci. 1 (12), 810-818.

DOI: $10.1021 / \mathrm{cn} 1000765$

Kpan J.D.A., Opoku B.K. y Gloria A. (2014). Heavy metal pollution in soil and water in some selected towns in Dunkwa-on-Off in District in the Central region of Ghana as a result of small-scale gold mining. J. Agric. Chem. Environ. 3 (2), 40-47.

DOI: 10.4236/jacen.2014.32006

Li, W.C. y Tse H.F. (2015). Health risk and significance of mercury in the environment. Environ. Sci. Pollut. Res. 22 (1), 192-201. DOI: 10.1007/s11356-014-3544-x

Loska K., Wiechuła D. y Korus I. (2004). Metal contamination of farming soils affected by industry. Environ. Inter. 30 (2), 159-165.

DOI: $10.1016 / \mathrm{S} 0160-4120(03) 00157-0$

Llanos W., Kocman D., Higueras P. y Horvat M. (2011). Mercury emission and dispersion models from soils contaminated by cinnabar mining and metallurgy. J. Environ. Monit. 13 (12), 3460-3468.

DOI: $10.1039 / \mathrm{c} 1 \mathrm{em} 10694 \mathrm{e}$

Marrugo-Negrete J., Olivero-Verbel, J., Ceballos E. y Benitez L. (2008a). Total mercury and methylmercury concentrations in fish from the Mojana region of Colombia. Environ. Geochem. Health. 30 (1), 21-30. DOI: $10.1007 / \mathrm{s} 10653-007-9104-2$

Marrugo-Negrete J., Benitez L.N. y Olivero-Verbel J. (2008b). Distribution of mercury in several environmental compartments in an aquatic ecosystem impacted by gold mining in northern Colombia. Arch. Environ. Contam. Toxicol. 55 (2), 305-316. DOI: $10.1007 / \mathrm{s} 00244-007-9129-7$

Matschullat J., Ottenstein R. y Reimann C. (2000). Geochemical background-Can we calculate it? Environ. Geol. 39 (9), 990-1000. DOI: 10.1007/s002549900084

McKeague J.A. y Wolynetz M.S. (1980). Background levels of minor elements in some Canadian soils. Geoderma. 24 (4), 299-307.

DOI: 10.1016/0016-7061(80)90057-9

Miller C.L., Watson D.B., Lester B.P., Lowe K.A., Pierce E.M. y Liang L. (2013). Characterization of soils from an industrial complex contaminated with elemental mercury. Environ. Res. 125, 20-29. DOI: 10.1016/j.envres.2013.03.013

MINAMBIENTE (2015). Sinopsis nacional de la minería aurífera artesanal y de pequeña escala. Documento. Ministerio de Ambiente y Desarrollo Sostenible, Bogotá, $72 \mathrm{pp}$.

Mmolawa K.B., Likuku A.S. y Gaboutloeloe G.K. (2011). Assessment of heavy metal pollution in soils along major roadside areas in Botswana. Afr. J. Environ. Sci. Technol. 5 (3), 186-196.
Müller G. (1981). Die Schwermetallbelastung der Sedimente des Neckars und seiner Nebenflusse: Eine Bestandsaufnahme. Chem-Ztg. 105, 157-164.

Niane B., Moritz R., Guédron S., Ngom P.M., Pfeifer H.R., Mall I. y Poté J. (2014). Effect of recent artisanal small-scale gold mining on the contamination of surface river sediment: Case of Gambia River, Kedougou region, southeastern Senegal. J. Geochem. Explor. 144 (Part C), 517-527. DOI: 10.1016/j.gexplo.2014.03.028

Odumo B., Carbonell G., Angeyo H.K., Patel J.P., Torrijos M. y Rodríguez M. (2014). Impact of gold mining associated with mercury contamination in soil, biota sediments and tailings in Kenya. Environ. Sci. Pollut. Res. 21 (21), 12426-12435.

DOI: $10.1007 / \mathrm{s} 11356-014-3190-3$

Olivero-Verbel J., Mendonza C. y Mestre J. (1995). Hair mercury levels in different occupational groups in Southern Bolivar (Columbia). Rev. Saúde Pública 29 (5), 376-379.

Olivero J. y Solano B. (1998). Mercury in environmental samples from a waterbody contaminated by gold mining in Colombia, South America. Sci. Total Environ. 217 (1-2), 83-89. DOI: 10.1016/S0048-9697(98)00175-2

Olivero-Verbel J., Johnson B. y Argüello E. (2002). Human exposure to mercury in San Jorge river basin, Colombia (South America). Sci. Total Environ. 289 (1-3), 41-47. DOI: 10.1016/S0048-9697(01)01018-X

Olivero-Verbel J., Caballero-Gallardo K. y Torres-Fuentes N. (2009). Assessment of mercury in muscle of fish from Cartagena Bay, a tropical estuary at the north of Colombia. Int. J. Environ. Health Res. 19 (5), 343-355. DOI: 10.1080/09603120902749090

Olivero-Verbel J., Caballero-Gallardo K. y MarrugoNegrete J. (2011). Relationship between localization of gold mining areas and hair mercury levels in people from Bolivar, north of Colombia. Biol. Trace Elem. Res. 144 (1), 118-132.

DOI: $10.1007 / \mathrm{s} 12011-011-9046-5$

Olivero-Verbel J., Young-Castro F. y Caballero-Gallardo K. (2014). Contaminación por mercurio en aire del distrito minero de San Martín de Loba en el departamento de Bolívar, Colombia. Rev. Int. Contam. Ambie. 30 (1), 7-13.

Olivero-Verbel J., Caballero-Gallardo K. y Turizo-Tapia A. (2015). Mercury in the gold mining district of San Martin de Loba, South of Bolivar (Colombia). Environ. Sci. Pollut. Res. 22 (8), 5895-5907.

DOI: $10.1007 / \mathrm{s} 11356-014-3724-8$

Poste A.E., Muir D.C.G., Guildford S.J. y Hecky R.E. (2015). Bioaccumulation and biomagnification of mercury in African lakes: The importance of trophic status. Sci. Total Environ. 506-507, 126-136.

DOI: $10.1016 /$ j.scitotenv.2014.10.094 
Qi X., Lin Y., Chen J. y Ye Y. (2000). An evaluation of mercury emissions from the chlor-alkali industry in China. J. Environ. Sci. 12, 24-30.

Rabee A.M., Al-Fatlawy Y.F., Najim A.H. y Nameer M. (2011). Using Pollution Load Index (PLI) and geoaccumulation index (I-Geo) for the assessment of heavy metals pollution in Tigris river sediment in Baghdad Region. J. Al-Nahrain University 14 (4), 108-114.

Sajn R. (2003). Distribution of chemical elements in attic dust and soil as reflection of lithology and anthropogenic influence in Slovenia. J. Phys. IV France 107, 1173-1176. DOI: 10.1051/jp4:20030509

Santos-Francés F., García-Sánchez A., Alonso-Rojo P., Contreras F. y Adams M. (2011). Distribution and mobility of mercury in soils of a gold mining region, Cuyuni river basin, Venezuela. J. Environ. Manage. 92 (4), 1268-1276. DOI: 10.1016/j.jenvman.2010.12.003

Shacklette H.T. y Boerngen J.G. (1984). Element concentrations in soils and other surficial materials of the conterminous United States [en línea]. https://pubs. usgs.gov/pp/1270/ 29/04/2017.

Sholupov S., Pogarev S., Ryzhov V., Mashyanov N. y Stroganov A. (2004). Zeeman atomic absorption spectrometer RA-915+ for direct determination of mercury in air and complex matrix samples. Fuel Process. Technol. 85 (6-7), 473-485.

DOI: $10.1016 /$ j.fuproc.2003.11.003

SIMCO (2017). Estadísticas 2013. Datos actualizados al I trimestre. Sistema de Información Minero Colombiano. Estadísticas, 1 p.

Taueg C., Sanfilippo D.J., Rowens B., Szejda J. y Hesse J.L. (1992). Acute and chronic poisoning from residential exposures to elemental mercury-Michigan, 1989-1990. J. Toxicol. Clin. Toxicol. 30 (1), 63-67. DOI: $10.3109 / 15563659208994446$

Tobías F.J., Bech J. y Algarra P.S. (1997). Establishment of the background levels of some trace elements in soils of NE Spain with probability plots. Sci. Total Environ. 206 (2-3), 255-265.

DOI: $10.1016 / \mathrm{S} 0048-9697(97) 80014-9$

Tomlinson D.L., Wilson J.G., Harris C.R. y Jeffrey D.W. (1980). Problems in the assessment of heavy metal levels in estuaries and the formation of a pollution index. Helgolander Meeresun. 33 (1), 566-575.

DOI: $10.1007 / \mathrm{BF} 02414780$

Tsubaki T. y Irukayama K. (1977). Minamata disease. Methylmercury poisoning in Minamata and Niigata, Japan. North-Holland Publishing, Amsterdam, 317 pp.

Wößmann W., Kohl M., Grüning G. y Bucsky P. (1999). Mercury intoxication presenting with hypertension and tachycardia. Arch. Dis. Child. 80 (6), 556-557.

DOI: 10.1136/adc.80.6.556

Xu J., Bravo A.G., Lagerkvist A., Bertilsson S., Sjöblom R. y Kumpiene J. (2015). Sources and remediation techniques for mercury contaminated soil. Environ. Int. 74, 42-53. DOI: 10.1016/j.envint.2014.09.007

Yaylali-Abanuz G. (2011). Heavy metal contamination of surface soil around Gebze industrial area, Turkey. Microchem. J. 99 (1), 82-92.

DOI: 10.1016/j.microc.2011.04.004

Yin R., Gu C., Feng X., Hurley J.P., Krabbenhoft D.P., Lepak R.F., Zhu W., Zheng L. y Hu T. (2016). Distribution and geochemical speciation of soil mercury in Wanshan Hg mine: Effects of cultivation. Geoderma 272, 32-38. DOI: 10.1016/j.geoderma.2016.03.003

Zhang H.H., Li F.B., Wu Z.F., Li D.Q., Xu D.R. y Yuan H.X. (2008). Baseline concentrations and spatial distribution of trace metals in surface soils of Guangdong Province, China. J. Environ. Qual. 37 (5), 1752-1760. DOI: $10.2134 /$ jeq2007.0531

Zhao F.J., McGrath S.P. y Merrington G. (2007). Estimates of ambient background concentrations of trace metals in soils for risk assessment. Environ. Pollut. 148 (1), 221-229. DOI: 10.1016/j.envpol.2006.10.041 